\title{
Topological evolution of Parker-unstable galactic magnetic fields under the influence of Coriolis force and magnetic reconnection
}

\author{
M. Hanasz ${ }^{1,2}$, K. Otmianowska-Mazur ${ }^{3}$, and H. Lesch ${ }^{1}$ \\ 1 University Observatory, München University, Scheinerstr. 1, 81679, Germany \\ e-mail: lesch@usm.uni-muenchen.de \\ 2 Toruń Centre for Astronomy, Nicolaus Copernicus University, 87148 Piwnice/Toruń, Poland \\ 3 Astronomical Observatory, Jagiellonian University, Krakow, Poland \\ e-mail: otmian@oa.uj.edu.pl
}

Received 6 September 2001 / Accepted 7 January 2002

\begin{abstract}
We investigate the influence of the Coriolis force and magnetic reconnection on the evolution of the Parker instability in galactic disks. We apply a model of a local gas cube, permeated by an azimuthal large-scale magnetic field and solve numerically resistive 3D MHD equations including the contribution of the Coriolis force. We introduce a current-dependent resistivity which switches the magnetic reconnection above a certain critical current density. We study the evolution of the magnetic field topology and the formation of large-scale poloidal magnetic fields from the initial azimuthal field. Our simulations demonstrate that the Parker instability leads to the formation of helically twisted magnetic flux tubes which are then agglomerated by magnetic reconnection forming, a significant poloidal magnetic field component on the scale of the whole cube. Such an evolution represents a kind of fast dynamo process as proposed by Parker (1992, ApJ 401, 137).
\end{abstract}

Key words. galaxies: ISM - magnetic fields - ISM: magnetic fields - MHD

\section{Introduction}

The problem of topological evolution of magnetic fields in conducting media is closely related to the amplification of the large-scale magnetic field by dynamo action. The idea of the dynamo is to use small-scale turbulent motions in the rotating medium in order to stretch and coherently twist small-scale magnetic loops. Such a chain of processes produces poloidal magnetic field flux from the azimuthal one and the subsequent differential rotation reproduces a toroidal magnetic field with some gain (e.g. Parker 1979 and references therein).

It turns out that the Parker instability is an ideal candidate to stretch the magnetic field lines and to produce coherently twisted loops under the influence of Coriolis force. It has been emphasized by Parker (1992) that the instability can lead to tangential discontinuities driving magnetic reconnection between adjacent magnetic lobes. Magnetic reconnection provides the required dissipation process by transforming small-scale magnetic structures into large-scale magnetic field configurations. The concept of Parker instability playing the driving role for a galactic dynamo has already been explored by Parker (1992),

Send offprint requests to: M. Hanasz, e-mail: mhanasz@astri.uni.torun.pl
Hanasz \& Lesch $(1993,1997,1998,2000)$ and Moss et al. (1999).

The aim of this paper is to show that the essential elements of Parker's (1992) fast dynamo model appear in 3D resistive MHD numerical simulations of the Parker Instability. In the next sections we formulate the problem, describe the setup of our numerical simulations, present results of the simulations and finally summarize our paper.

\section{Formulation of the problem}

In the present paper we focus our attention on the issue of 3D evolution of the Parker instability in the presence of rigid rotation and fast magnetic reconnection. We limit our attention to the case of rigid rotation in order to study clear effects of the Parker instability (as Brandenburg \& Schmitt 1998). A complementary study including the effects of differential rotation will be published elsewhere. The differential rotation alone (i.e. without the vertical stratification and buoyancy) is responsible for the magneto-rotational instability (see the review by Balbus \& Hawley 1998) through the tidal forces that are absent in the case of rigid rotation. The mixed effect of buoyancy and magneto-rotational instabilities in astrophysical disks has already been studied in some aspects by Foglizzo \& Tagger (1994, 1995), Hawley et al. (1996), 
Terquem \& Papaloizu (1996), Hanasz \& Lesch (1997), Machida et al. (2000) and Kim et al. (2000). One should note however that the coupling between the Parker and the Balbus-Hawley instability is rather complex, therefore we shall skip the effects of shear in this contribution.

Our initial equilibrium state is an exponentially stratified disk as proposed by Parker (1966) and frequently used by other authors (see e.g. Kim et al. 1998, 2001). These authors perform numerical simulations of the Parker instability for a similar physical setup, however they focus on different issues. The equilibrium state is characterized by an uniform vertical gravity, an isothermal equation of state and a sound speed which is constant across the disk. The fixed ratio of the magnetic pressure to the gas pressure is given by $\alpha=p_{\text {mag }} / p_{\text {gas }}$. In a Cartesian reference frame with coordinates $x, y, z$ corresponding locally to the radial, azimuthal and vertical coordinates in the disk, the assumed initial magnetic field is purely azimuthal, $\boldsymbol{B}_{0}=B_{0}(z) \boldsymbol{e}_{y}$ and the dependence of equilibrium quantities on $z$ is

$\frac{p_{0}(z)}{p_{0}(0)}=\frac{n_{0}(z)}{n_{0}(0)}=\frac{B_{0}^{2}(z)}{B_{0}^{2}(0)}=\exp \left(\frac{-z}{H}\right)$

with the midplane particle density $n_{0}(0)=1 \mathrm{~cm}^{-3}$, the isothermal sound speed $c_{\mathrm{s}}=7 \mathrm{~km} \mathrm{~s}^{-1}$, vertical gravitational acceleration $g=-2 \times 10^{-9} \mathrm{~cm} \mathrm{~s}^{-2}, \alpha=1.0$ and a vertical scaleheight $H=(1+\alpha) c_{\mathrm{s}}^{2} /|g| \simeq 150 \mathrm{pc}$. As an instructive example we use throughout the paper the angular velocity of the galactic rotation of the Solar orbit $\Omega=0.025 \mathrm{Myr}^{-1}$. Likewise all other parameters are typical for the contemporary Solar neighborhood.

The chosen value of $\alpha=1.0$ corresponds to the magnetic field of $4.5 \mu \mathrm{G}$ at the galactic midplane, which is larger than the observed regular magnetic field of the order of 1.8-4 $\mu \mathrm{G}$ (Beck 2001). We use such a strong magnetic field in the present experiments because the growth rate of the Parker instability for smaller values of $\alpha$ is unrealistically small due to the lack of cosmic rays in the present model. Therefore the strong magnetic field in our numerical experiments partially compensates the contribution of the cosmic ray component to the buoyancy. Our aim is to study Parker's idea of the generation of poloidal magnetic field by the buoyancy instability in the presence of Coriolis force and for this purpose the relatively strong initial magnetic field corresponding to $\alpha=1$ seems to serve quite well. Similar effects would appear with a weak magnetic field and a strong cosmic ray contribution, since both components (cosmic rays and magnetic fields) are massless but add to the pressure, i.e. enhance the buoyancy force. The assumption of $\alpha=1$ implies that we deal with a contemporary magnetic field. We note however that a fast magnetic field amplification is necessary to explain the large pitch angles of magnetic fields in nearby galaxies (see e.g. Chyży et al. 2000; Otmianowska-Mazur et al. 2000).

We do not study the timescale nor the lengthscale of the most unstable Parker unstable modes, since this cannot be done reliably without the contribution of cosmic rays (see Hanasz \& Lesch 2000). We do not take explicitly into account small scale turbulence nor magnetic field meandering in the galactic disk. The last two effects appear to be important for the geometry of the most unstable modes. Without them, the most unstable modes are those that have infinitely large transverse horizontal wavenumber. Under the influence of turbulence and a meandering magnetic field, the transverse wavenumbers of the most unstable modes become finite (Parker 1967; Zweibel \& Kulsrud 1975; Lachieze-Ray et al. 1980; Parker \& Jokipii 2000). To examine the role of transverse wavenumbers we construct random velocity perturbations as superpositions of trigonometric components with random phases and vary the number of harmonic components of perturbations in order to obtain different transverse dominating wavenumbers in different simulation runs.

The perturbations applied are of two types. The first one is the unimodal perturbation of the vertical velocity $v_{z}$ of the form

$v_{z}(x, y, z)=v_{0} \cos \left(\frac{2 \pi n_{x} x}{L_{x}}\right) \cos \left(\frac{2 \pi y}{L_{y}}\right) \sin \left(\frac{\pi z}{H}\right)$

for $0 \leq z \leq H$, and $v_{z}(x, y, z)=0$ for $H \leq z \leq L_{z}$. $v_{0}=0.1 \mathrm{~km} \mathrm{~s}^{-1}$ is the initial perturbation amplitude; $L_{x}$, $L_{y}, L_{z}$ are the physical sizes of the simulation domain. That means that there is a single spatial period of perturbation in the computational domain along the magnetic field and $n_{x}=5$ periods in the direction perpendicular to the magnetic field.

The second type of perturbation is more complex. It is composed of a superposition of different higher harmonics of the fundamental periodic waves in the lower part of the computational volume, $0 \leq z \leq H$ as above. The perturbations of this type are given by the formula

$$
\begin{aligned}
v_{z}(x, y, z)= & \sum_{i_{x}, i_{y}, i_{z}=1}^{n_{x}, n_{y}, n_{z}} \frac{v_{0}}{n_{x} n_{y} n_{z}} \cos \left(\frac{2 \pi i_{x} x}{L_{x}}+\phi_{i_{x}}\right) \\
& \times \cos \left(\frac{2 \pi i_{y} y}{L_{y}}+\phi_{i_{y}}\right) \sin \left(\frac{\pi i_{z} z}{H}+\phi_{i_{z}}\right)
\end{aligned}
$$

where $n_{x}, n_{y}$ and $n_{z}$ are the numbers of harmonic components of the $x, y$ and $z$ direction. $\phi_{i_{x}}, \phi_{i_{y}}$ and $\phi_{i_{z}}$ are random phases. The limitation of perturbations to $z \leq H$ is motivated by the assumption that the stellar activity of the disk (e.g. supernova explosions stellar winds or cosmic ray input) initiates the instability.

\section{Magnetohydrodynamic equations and plasmaphysical arguments}

In order to describe the isothermal evolution and the rigid rotation of the system we apply the following set of resistive MHD equations

$$
\frac{\partial \rho}{\partial t}+\nabla \cdot(\rho \boldsymbol{v})=0
$$

$\frac{\partial \boldsymbol{v}}{\partial t}+(\boldsymbol{v} \cdot \boldsymbol{\nabla}) \boldsymbol{v}=-\frac{1}{\rho} \boldsymbol{\nabla}\left(p+\frac{B^{2}}{8 \pi}\right)+\frac{\boldsymbol{B} \cdot \boldsymbol{\nabla} \boldsymbol{B}}{4 \pi \rho}-2 \boldsymbol{\Omega} \times \boldsymbol{v}$ 
$\frac{\partial \boldsymbol{B}}{\partial t}=\boldsymbol{\nabla} \times[\boldsymbol{v} \times \boldsymbol{B}-\eta \boldsymbol{j}]$

where $\boldsymbol{j}=\boldsymbol{\nabla} \times \boldsymbol{B}$ is the current density and the other symbols have their usual meaning.

We use the concept of an anomalous resistivity which depends on the current density (Ugai 1992; Biskamp 1997; Tanuma et al. 1999, 2001; Konz et al. 2000)

$\eta(j)=\eta_{2}\left(j^{2}-j_{\text {crit }}^{2}\right)^{1 / 2} \Theta\left(j^{2}-j_{\text {crit }}^{2}\right)$,

where $\eta_{2}$ is a constant coefficient, $j_{\text {crit }}$ denotes the critical current density above which the resistivity switches on and $\Theta$ is the Heaviside step function. This ansatz for the resistivity needs some explanation. In general, the electrical resistivity describes the drag forces onto charged particles which carry the electrical current density $j$. In a collisionless plasma the drag force is not due to Coulomb collisions but is provided by plasma fluctuations which contain oscillating electromagnetic fields. Those oscillations influence the particles motion and thus provide the resistivity inside the current sheet (e.g. Treumann \& Baumjohann 1997). From a plasma physical point of view the energy source for the fluctuations is given by the relative motion of positive and negative charges, respectively. In other words, if the negative charges exceed a certain critical drift velocity with respect to the positive charges, i.e. if a critical current density is exceeded plasma waves are excited which drag the current carrying particles by wave-particle scattering (e.g. Sagdeev 1979).

Whenever an ideal magnetized plasma in which no Coulomb collisions are present is disturbed externally the current density will increase until it reaches the temperature-dependent critical value above which plasma fluctuations are excited and provide an anomalous resistivity. Thus, in any collisionless, magnetized astrophysical plasma exposed to some disturbance, magnetic reconnection will relax the distorted magnetic field by dissipation of magnetic energy into heat or particle acceleration via topological changes (e.g. Priest 1987). In this respect our investigation is of general interest for any magnetic field in a collisionless plasma. This chain of plasma response reactions to an external disturbance is a quite natural one for a system which is externally distorted. Unsaturated external forces energetically excite the system and drive it into a sort of crisis, i.e. away from an equilibrium state. This externally supported nonequilibrium state now is the source of relaxation processes reaching either a new equilibrium state or the old equilibrium. Reconnection presents such an relaxation answer of a magnetized agitated nonequilibrium plasma to an external driver. Above a critical state, reconnection sets in and relaxes the magnetic field to a configuration of lower energy.

The most important aspect of magnetic reconnection concerns its localized character. The condition $j \geq j$ crit is only fulfilled in localized regions of the plasma. Thus the topological changes will appear where reconnection could proceed. This is contrary to the diffuse resistivity present everywhere in a plasma by the small but finite
Coulomb resistivity, which drives the global diffusion of the magnetic field. Formally, magnetic reconnection belongs to the term $\nabla \times\left(\eta_{\text {anom }} \nabla \times \boldsymbol{B}\right)$, whereas diffusion is related to $\eta_{\text {Coulomb }} \Delta \boldsymbol{B}$, where $\eta_{\text {anom }}$ denotes the spatial dependent anomalous current-driven resistivity and $\eta_{\text {Coulomb }}$ is the scalar Coulomb resistivity which depends only on the plasma temperature (Braginskii 1965) and thus is much more smoothly distributed than the localized current-driven resistivity.

The above prescription of the resistivity allows for a useful parameterization of the magnetic reconnection. We emphasize that within our numerical setup (next section) we do not resolve the real small scale current sheets in turbulent medium, but only describe large-scale planar magnetic structures in which small-scale reconnection sheets are localized and produce the global effect of evolving magnetic field topology. Unfortunately, the spatial scales typical for real current sheets expected in the interstellar medium are several orders of magnitude smaller than typical sizes of grid cells typically adopted for numerical simulations of the Parker instability. Thus, the only way to incorporate magnetic reconnection is to parameterize the sub-scale reconnection processes (Ugai 1992; Tanuma et al. 1999; Konz et al. 2000). One possible parameterization is applied in the present paper, however the final results are almost independent on the choice of parameters, so we expect that the results presented in the next sections are sufficiently general.

Our assumption of an isothermal evolution is equivalent to a redundant energy equation. The relation between pressure and density is given by $p=c_{\mathrm{si}}^{2} \rho$, where $c_{\mathrm{si}}^{2}=k_{\mathrm{B}} T / m$ is the isothermal sound speed. The isothermal assumption, whether it is applicable or not, implies that thermal equilibrium is maintained with an external infinite reservoir of heat of a fixed temperature $T$. It means also that the heat is transported over the system on a timescale significantly shorter than the dynamical timescale. If resistivity is taken into account in the adiabatic case, the resistive terms appear in the induction equation and in the energy equation. Our isothermal assumption requires an instantaneous heat transport from the reconnection region into the surrounding medium. In this case the only signature of the reconnection is the change of topology of magnetic field lines and not the heating of the reconnection region.

One can estimate the timescales of radiative cooling of the highly ionized interstellar medium for temperatures in the range of $10^{2}-10^{4} \mathrm{~K}$ and hydrogen number densities in the range $10^{-3}-1 \mathrm{~cm}^{-3}$ typical for $|z| \leq 1 \mathrm{kpc}$ above the galactic midplane. The cooling timescale is given by the formula

$t_{\text {cool }}=\frac{n k_{\mathrm{B}} T}{n^{2} \mathcal{L}(T)}$

where $\mathcal{L}(T)$ denotes the cooling function (Dalgarno \& McCray 1972). One can estimate the cooling time separately for different gaseous phases of the interstellar medium assuming a specific model of vertical gas 
distribution (see Ferriere 1998a for the case of Milky Way). In case of warm ionized medium the cooling time is about 1-3 Myr, which is one order of magnitude shorter than the growth time of the Parker instability. The relatively short cooling time of the warm ionized medium means that if we want to omit the full complexity of heating and cooling processes, the isothermal approximation is as reasonable as an adiabatic one.

The cooling times of colder and denser constituents are typically shorter or equal to $10^{5} \mathrm{yr}$. The cooling time of the hot medium is much longer, but this phase occurs with a relatively small filling factor and low density (Ferriere 1998b), therefore its presence is neglected in our present considerations. However, it is quite obvious that the hot phase may play a role, especially at large distances from the galactic midplane, so it should be included in future studies of the Parker instability.

On the other hand we are aware that resistive processes like magnetic reconnection will locally increase the temperature on very small timescales. We note that magnetic reconnection has been invoked as an additional heat source for ISM (see e.g. Parker 1992; Raymond 1992; Kahn \& Brett 1993; Birk et al. 1998; Hanasz \& Lesch 1998; Tanuma et al. 1999, 2001) and is mentioned by Reynolds et al. (1999) as a possible heat source in regions of electron densities below $0.1 \mathrm{~cm}^{-3}$. However, since these heated regions are tiny in comparison to the spatial volumes occupied by the flux tubes we consider only the large scale heating and cooling properties. In other words, isothermality is guaranteed as long as the large scale volume can cool faster than the material ascends from the disk via unstable magnetic flux tubes.

Thus, in our simulations magnetic reconnection does not increase the overall temperature of the gas. The only effect it has is to change the magnetic topology. The reconnection rate in the interstellar medium is supposed to be of the order of a few percent of the Alfven speed (Kahn \& Brett 1993).

\section{Numerical simulations}

To perform the simulations we use the ZEUS-3D MHD code (see Stone \& Norman 1992a, 1992b) with our own modifications necessary to investigate the described problem. Concerning the standard options of the code we use the full 3D performance of the code in Cartesian coordinates, choosing the isothermal equation of state (ISO) and the method of characteristics (HSMOC) algorithm for the integration of magnetic field. In addition to the standard features of the code we have introduced the vertical uniform gravity, the Coriolis force in Cartesian coordinates and resistivity. Concerning the numerical algorithm we introduced the resistivity in a way similar to that by Fleming et al. (2000). First the HSMOC technique is used to update the first term on the R.H.S of the induction Eq. (6) and then the constraint transport (CT) is used to update the magnetic flux according to the resistive term $-\eta \boldsymbol{j}$ in (6). On the physical side we have introduced the current-dependent resistivity according to the formula (7) instead of the constant resistivity adopted by Fleming et al. (2000).

We performed a series of more than 20 numerical simulations of the Parker instability applying different perturbations resulting from the formulae (2) and (3). The major simulations were done with the resolution of $120 \times 90 \times 240$ zones (not including the ghost zones) in the $x, y$ and $z$ directions, respectively. To check the effect of the finite grid resolution we have repeated the same simulations with the resolution of $60 \times 90 \times 120$ grid cells, however these simulations were different only in minor details compared to the high resolution cases. Thus, we describe in the next sections only the simulations with high resolution runs. For all the simulations we have applied the Cartesian computational domain of physical sizes of $L_{x}=600 \mathrm{pc}, L_{y}=1800 \mathrm{pc}$ and $L_{z}=600 \mathrm{pc}$ (note that $L_{y} \simeq 12 H$ is the wavelength of the most unstable mode of the Parker instability in our case). We used the higher grid resolution in the direction transverse to the initial magnetic field $\boldsymbol{B} \| \boldsymbol{y}$ because test simulations have shown that the gradients of physical quantities (mainly because of the tangent discontinuities in $\boldsymbol{B}$ ) appeared to be much larger in the $x$ and $z$ directions than in the $y$ direction.

In half of our high resolution runs we had no anomalous resistivity, but only the unavoidable homogeneously distributed numerical resistivity. The other $50 \%$ adopted a localized resistivity characterized by the coefficient $\eta_{2}=100$ and the critical current density $j_{\text {crit }}=0.4$ for the high resolution runs. These parameters determine the magnitude of resistivity defined by Eq. (7), which is localized in regions (current sheets) where current density exceeds $j_{\text {crit }}$. The critical current density was adopted experimentally to make magnetic reconnection effective as soon as the current sheets form. Our numerical experiments have shown that the overall behavior of the system is only weakly dependent on the particular choice of these parameters, thus we are not going to discuss the differences implied by the variation of these parameters.

We applied periodic boundary conditions in the domain walls perpendicular to the magnetic field and reflection boundary conditions in all the domain walls parallel to the unperturbed magnetic field. The boundary conditions influence the regions close to the vertical walls parallel to the original magnetic field slightly, but the effect is not relevant for the internal parts of the simulation volume.

With this setup we performed a series of simulations. In the following we discuss in detail only the most representative ones.

Our main simulations A0 and A1 are based on a very simple initial perturbation given by formula (2). These experiments allow for an easy demonstration of the main qualitative effects of the physical evolution of the system. The complementary simulations of a more complex and more general form are constructed according to formula (3). The most important difference between various perturbations of this type is given by the numbers 
Table 1. Input parameters for chosen models.

\begin{tabular}{cccc}
\hline \hline Run & $\begin{array}{c}\text { Perturb. } \\
\text { Eq. No. }\end{array}$ & $n_{x} \times n_{y} \times n_{z}$ & $\begin{array}{c}\text { Resis- } \\
\text { tivity }\end{array}$ \\
\hline A0 & $(2)$ & - & $\mathrm{N}$ \\
A1 & $(2)$ & - & $\mathrm{Y}$ \\
B0 & $(3)$ & $3 \times 3 \times 1$ & $\mathrm{~N}$ \\
B1 & $(3)$ & $3 \times 3 \times 1$ & $\mathrm{Y}$ \\
C1 & $(3)$ & $5 \times 5 \times 5$ & $\mathrm{Y}$ \\
D1 & $(3)$ & $10 \times 5 \times 5$ & $\mathrm{Y}$ \\
\hline
\end{tabular}

$n_{x}, n_{y}$ and $n_{z}$ of the harmonic components entering the perturbation with random phases $\phi_{i_{x}}, \phi_{i_{y}}, \phi_{i_{z}}$.

The Col. 3 in the Table 1 informs about the number of harmonic components of the perturbation in each direction for a particular run.

\subsection{The ideal cases}

After imposing the initial velocity perturbation onto a gravitationally stratified state the unstable modes of the Parker instability start to grow in the familiar way forming waves on the initially horizontal magnetic field (see Fig. 9a). As soon as the vertical magnetic field deformations develop sufficiently the ionized gas slides down along the magnetic field leading to gas condensations in magnetic valleys. The upper parts of field lines devoided of heavy gas become lighter and rise due to the buoyancy force. The horizontal motions of gas are influenced by the Coriolis force.

The effect of Coriolis force visible as the strong counterclockwise twist of magnetic lines (up to 90 degrees) at gas condensations is presented in Fig. 1, where we show a horizontal cut at lower part of the computational volume for the run B0 at $t=430 \mathrm{Myr}$, which is a superposition of different modes according to the formula (3) (see Table 1). The grey plot in Fig. 1 represents the gas density perturbation $\Delta \rho / \rho_{0}=(\rho(t, \boldsymbol{r})-\rho(0, \boldsymbol{r})) / \rho(0, \boldsymbol{r})$, where the dark and light shades denote regions underdense and overdense with respect to the initial equilibrium density.

The evolution of the vertical structure of perturbations is demonstrated in Fig. 2, where we present cuts in the plane $x z$ through the computational volume for the run A0. In this run we have applied a perturbation representing a single mode which is prepared according to the formula (2).

The shades in Figs. 2 give the modulus of current density. Arrows indicate the magnetic field projected onto the $x z$-plane (perpendicular to the initial magnetic field direction). Due to the Coriolis force the rising lines of magnetic field are twisted opposite to the large scale rotation. Lower parts (see also Fig. 1) are twisted in the sense of galactic rotation. The same effect of the Coriolis force on the shape of magnetic field lines is also visible in Fig. 9a, where we show magnetic field lines resulting from the spatial integration of magnetic field vectors. The starting points of all lines are placed at $y=0 \mathrm{pc}, z=50 \mathrm{pc}$ and span the full range of the $x$ coordinate.

The gas flows down along the magnetic field lines forming thin vertical sheets in between the magnetically dominated regions and collects in long gas filaments. These filaments are partially supported by the twisted lower parts of the field lines. Dense sheets of gas coincide with current sheets separating regions of the opposite polarity of the vertical magnetic field components. We show this effect in Figs. 6 and 7 for the resistive runs $\mathrm{C} 1$ and D1 which are discussed in the next subsection. The current sheets form at the advanced evolutionary stages of the Parker instability. Up to $t=300 \mathrm{Myr}$ the maximum current density in the computational volume is small for all runs (corresponding to the large scale horizontal stratification of magnetic field) and practically constant, $j=|\boldsymbol{j}| \leq 0.05$ in normalized units. After that time $j$ starts to grow rapidly. In the absence of a physical resistivity for the run A0 $j=0.11$ at $t=350 \mathrm{Myr}, j=0.31$ at $t=400 \mathrm{Myr}$, $j=0.70$ at $t=450 \mathrm{Myr}$ and $j=0.58$ at $t=500 \mathrm{Myr}$. The saturation of the current around $t=450 \mathrm{Myr}$ is caused by the limited grid resolution - for a given jump of magnetic field at the contact discontinuity in between the neighboring grid cells the magnitude of current $\boldsymbol{j}=\operatorname{curl} \boldsymbol{B}$ is determined by the cell size. The effect of numerical resistivity is noticeable in Fig. $2 \mathrm{~b}$ at $t=500 \mathrm{Myr}$ where some topological changes of magnetic field start to occur. This process is however apparently weaker than the effect of current dependent anomalous resistivity described in the next subsection.

\subsection{The resistive cases}

In the presence of a current dependent resistivity the evolution of the system changes. As soon as the current density exceeds the critical current, the resistivity starts to operate in current sheets.

Results for different resistive runs are shown in Figs. 3 (A1), 5 and 4 (B1), 6 (C1), 7 (D1) and 9b, c (A1). In Fig. 3, we show arrows of the magnetic field projected on the $x z$-plane at $y=0 \mathrm{pc}$ overlaid on the image of the current density for run A1 which is the resistive counterpart of the run A0 based on the unimodal perturbation and described in the previous subsection. Comparing the magnetic field geometry at $t=500$ with the state of $t=400 \mathrm{Myr}$ in Fig. 2a we note the visible effects of the magnetic reconnection which operates in five vertical current sheets (the lightest regions in the current density distribution). The result of magnetic reconnection is the change of the topology of magnetic field. The evolution from $t=400 \mathrm{Myr}$ to $t=500 \mathrm{Myr}$ can be described as a transformation from closely packed magnetic structures (lobes) having oppositely directed vertical components of the magnetic field toward the system of helical flux tubes. The helical magnetic field component of these flux tubes is seen in Fig. 3a. The magnetic field lines originating at the same set of starting points as in the non-resistive case A0 


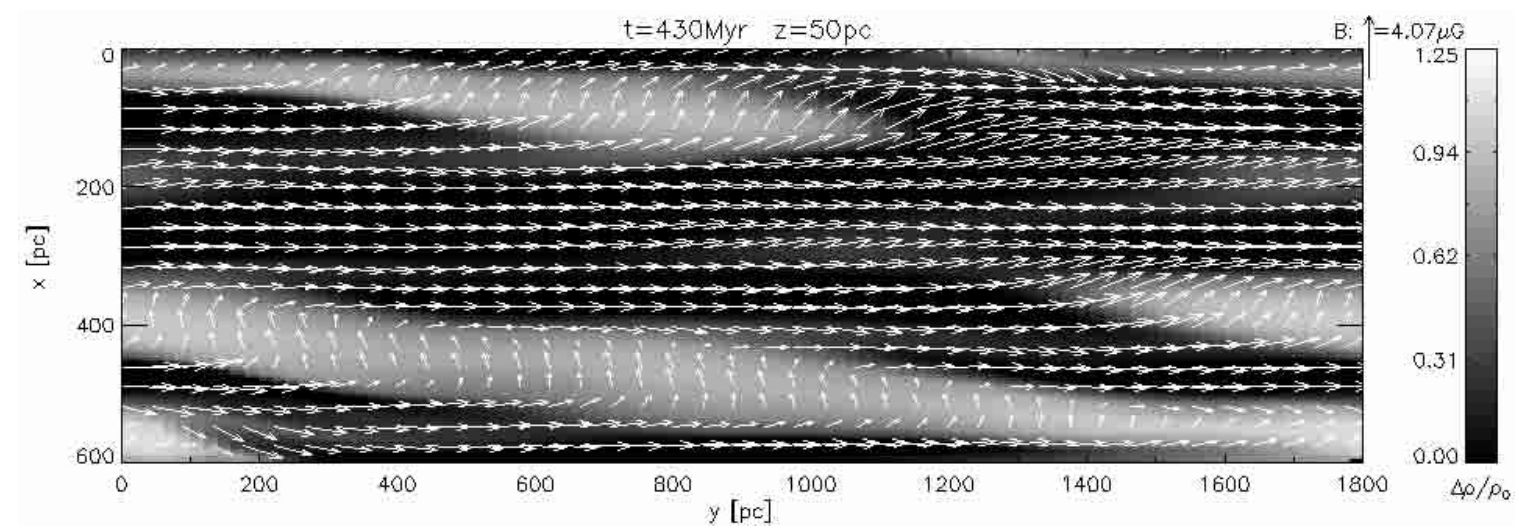

Fig. 1. A horizontal cut through the computational volume is shown for the model B0 at $t=430$ Myr for $z=50$ pc. The gray shades represent the gas density perturbation $\Delta \rho / \rho_{0}$, where dark and light shades denote regions underdense and overdense with respect to the initial equilibrium density. Arrows represent the magnetic vectors projected onto the $x y$-plane.
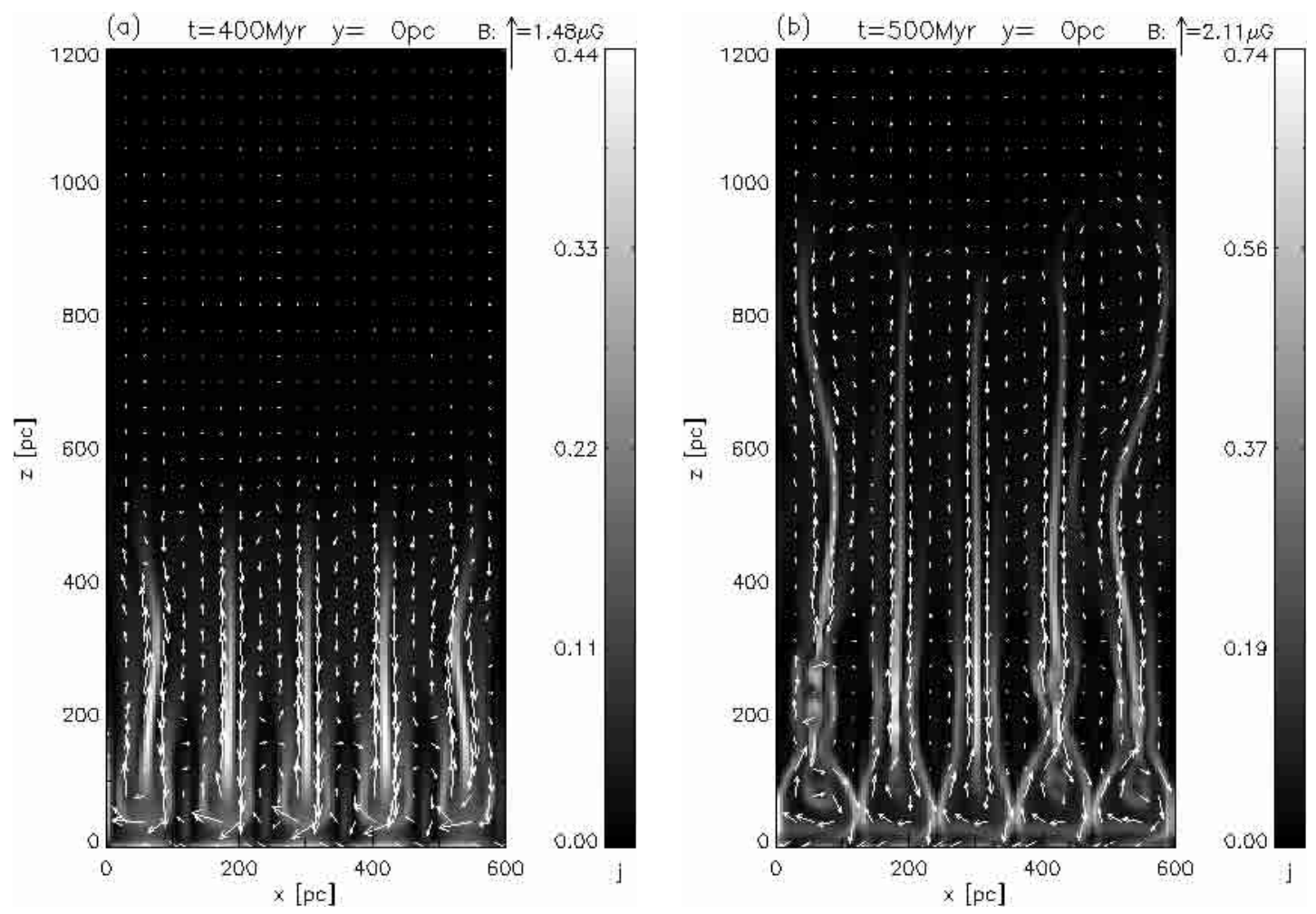

Fig. 2. The $x z$-cuts at $y=0 \mathrm{pc}$ present the poloidal component of the magnetic field projected onto the background of the current density for $t=400 \mathrm{Myr} \mathbf{a}$ ) and $t=500 \mathrm{Myr} \mathbf{b}$ ) in the ideal case (model A0). The light and dark grey shades denote respectively regions with high current (current sheets) and low current values.

are shown in Fig. 9b. Apparently the twisted flux tubes extend over the full length of the simulation box, i.e. over the full wavelength of the perturbed mode of the Parker instability. The centers of the tubes contain filaments of gas collected before reconnection occurred as described in the previous subsection (see e.g. Fig. 6b). The gas filaments are additionally compressed by the toroidal magnetic field. Moreover, the tubes are inclined with respect to the initial azimuthal direction parallel to the $y$-coordinate.

As we can see in Fig. 3a the tubes are closely packed in some cases and the helical magnetic field component of adjacent tubes gives rise to a next generation of contact discontinuities in magnetic field. Magnetic reconnection starts to act between neighboring flux tubes. The flux tubes merge and thus produce a large-scale magnetic field in the $x$-direction which is seen at $t \simeq 700 \mathrm{Myr}$ (see Figs. 3b, $4 \mathrm{~b}$ and $9 \mathrm{c})$.

The overall evolution of the system is very similar if one takes into account more general initial perturbations as given by formula (3). In the case of all resistive runs the evolution of perturbations proceeds through the formation of a system of almost vertical current sheets. The number of current sheets increases with the growing number of harmonics (preferentially in the $x$ direction) in the initial perturbation. It has been shown by Parker (1967) that in the absence of turbulence, the most unstable are 

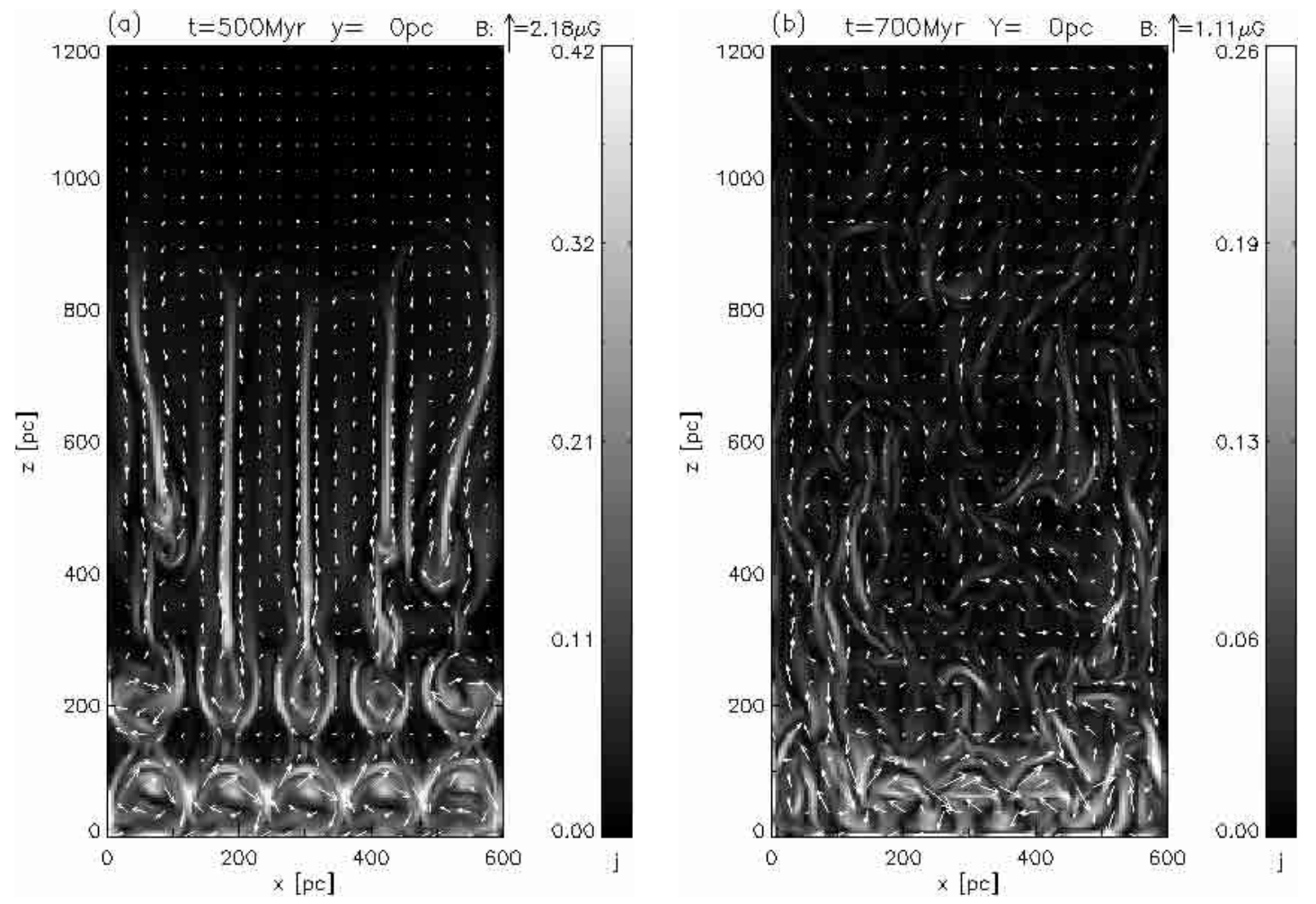

Fig. 3. Poloidal component of magnetic field on the background of current density in the resistive case (model A1). Magnetic reconnection converts the structures developed before $t=400 \mathrm{Myr}$ into a family of helical flux tubes clearly visible at $t=$ $500 \mathrm{Myr} \mathbf{a}$ ). Later on the tubes merge producing a new large scale poloidal magnetic field at $t=700 \mathrm{Myr} \mathbf{b}$ ).
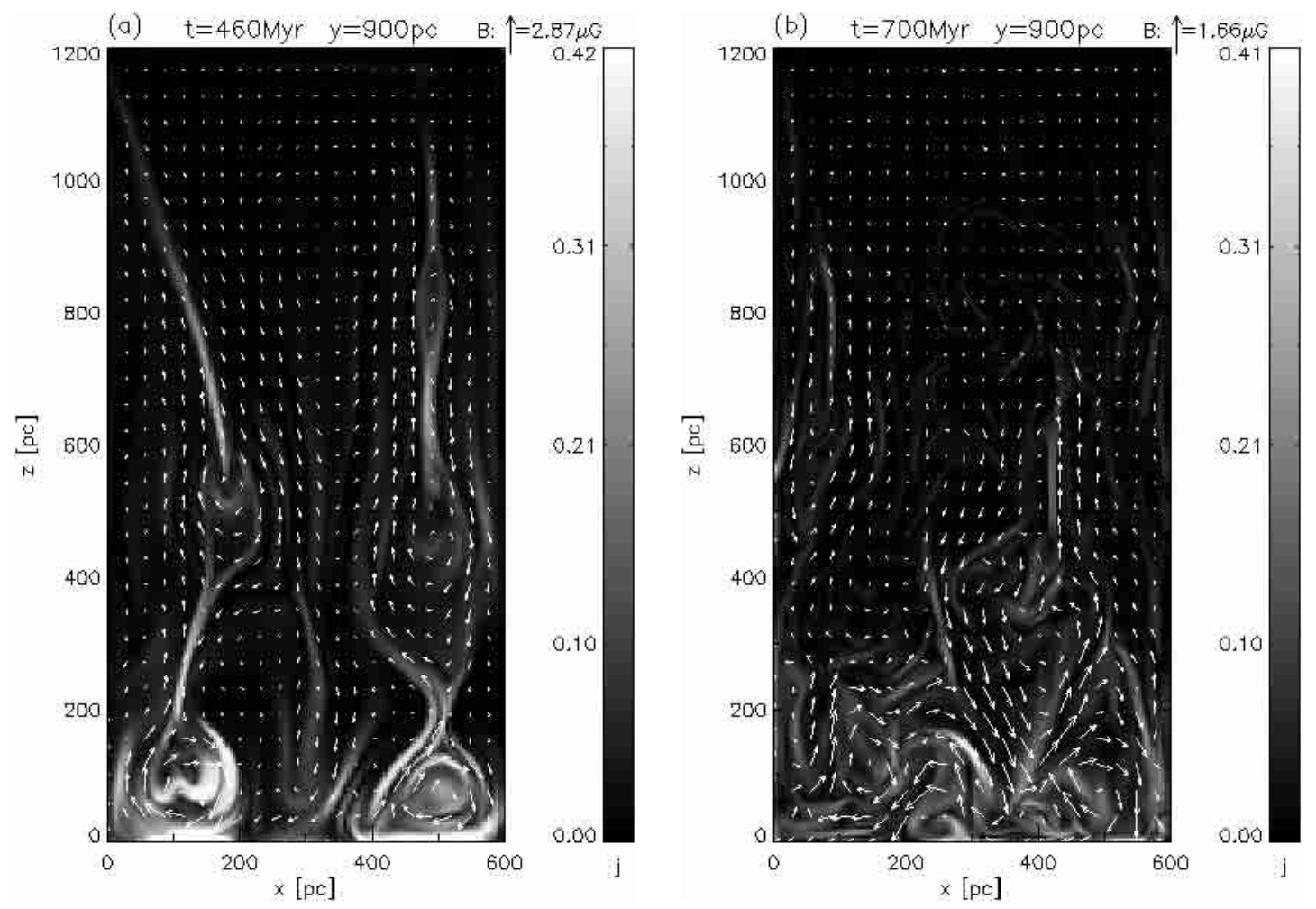

Fig. 4. Vertical cuts in the plane $x z$ and $y=900 \mathrm{pc}$ for the run B1. Arrows represent the magnetic vectors and shades indicate the current density. a) The helical magnetic field surrounding the elongated gas condensations is seen at lower parts of the figure. b) After some time evolution different helical structures merge to form a large scale magnetic in the $x$ direction at $t=700$ Myr. 


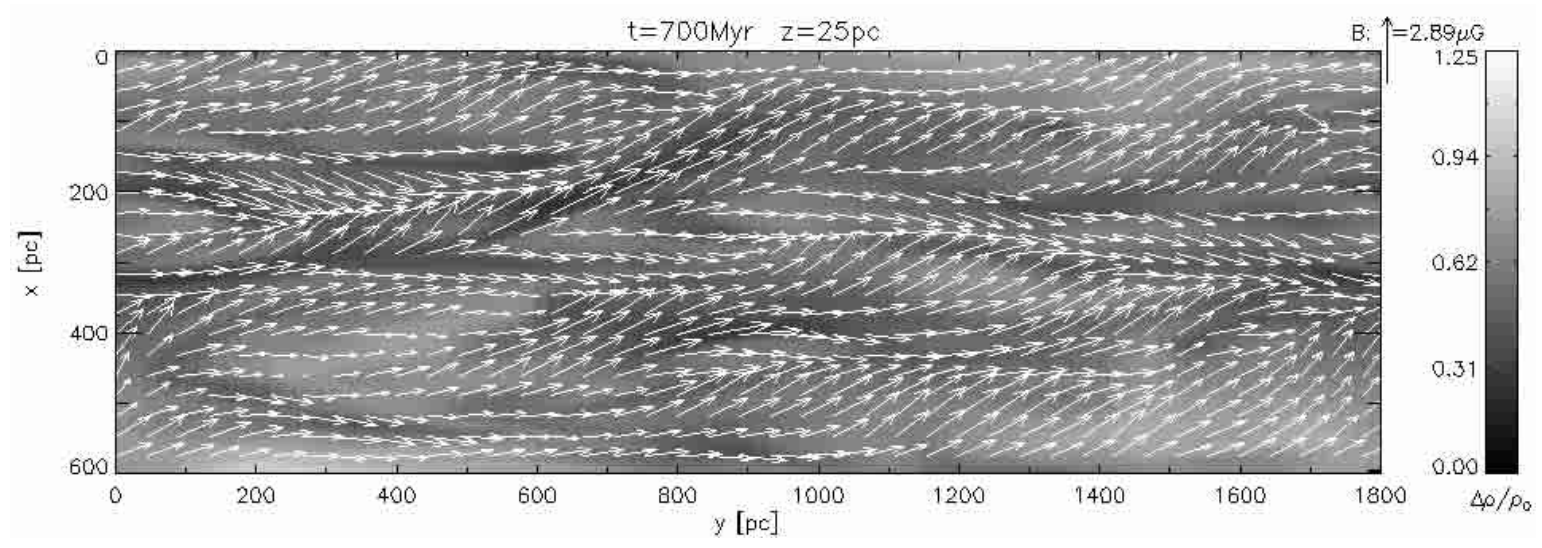

Fig. 5. The cut in the plane $x y$ at $z=25$ pc same as Fig. 4 for the run B1 at $t=700$ Myr. The gas distribution is more smooth than that shown in Fig. 1 for the run B0 at $t=430$ Myr and the twisted magnetic field spread out over the shown gas layer.
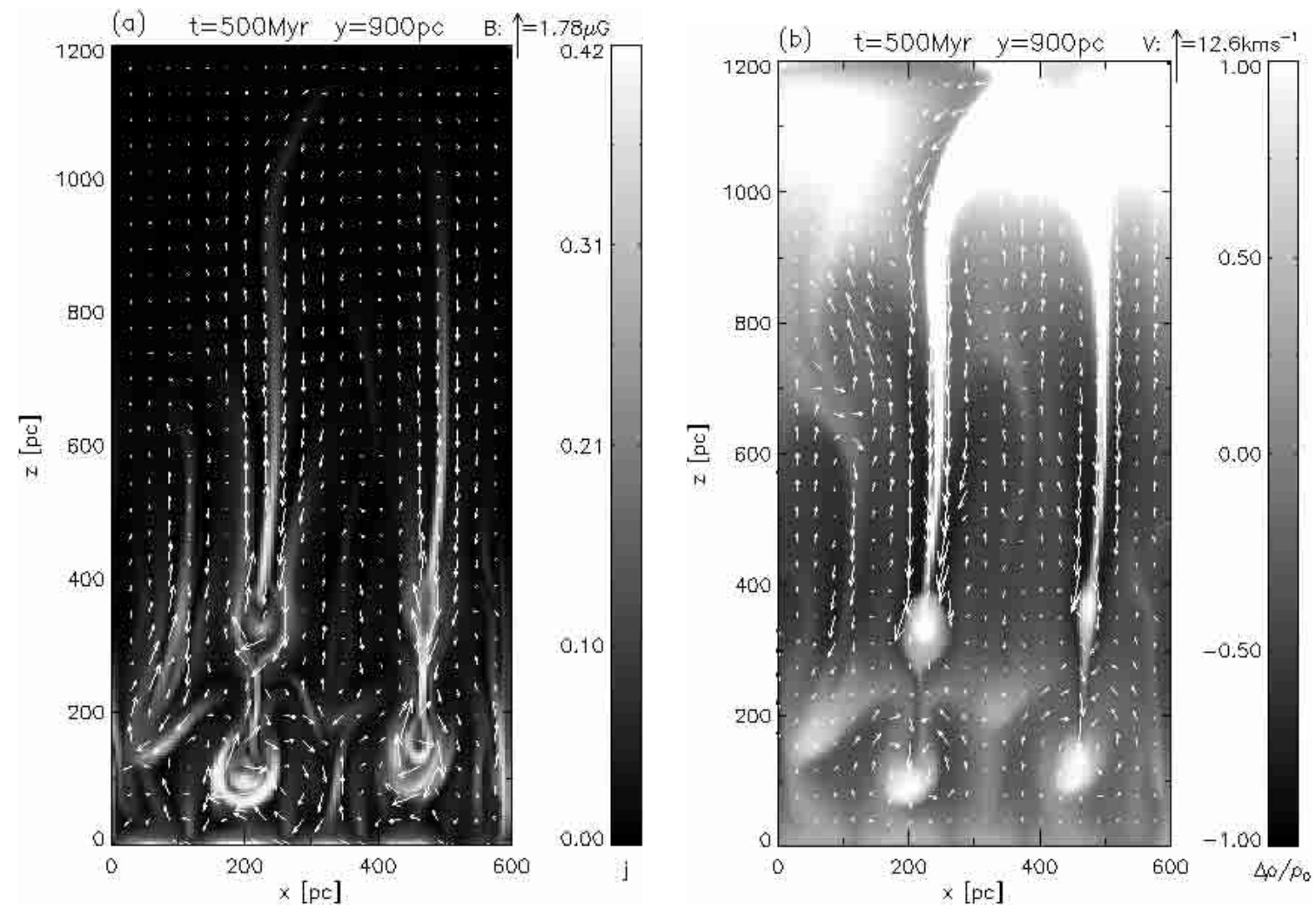

Fig. 6. Vertical cuts in the plane $x z$ and $y=900 \mathrm{pc}$ for the run $\mathrm{C} 1$ at $t=500$ Myr. Arrows represent magnetic field a) and velocity b). Shades indicate the current density a) and mass density $\mathbf{b}$ ).

the mode of the highest horizontal transverse wavenumbers. The more recent studies by Parker \& Jokipii (2000) demonstrated that in the presence of meandering or turbulent magnetic field these modes are stabilized depending on the strength of the nonuniform magnetic field component. In the present paper we intend however to examine different cases of small, intermediate and large horizontal transverse wavenumbers.

Since different harmonics contribute with the same amplitude to our initial perturbation, the fastest growing modes with the shortest wavelength in the horizontal direction perpendicular to the initial magnetic field start to dominate very soon. Looking at the vertical cuts of our computational box (Figs. 2a, 4a, 6a, 7a, we note the number of vertical current sheets is usually smaller than the number of harmonics $n_{x}$ in the $x$ direction. This is because the spatial randomness of our perturbations implies that the current sheets appear also randomly in time. For instance, the run $\mathrm{C} 1$ is perturbed by $n_{x}=5$ harmonic components in the $x$-direction, but only two vertical current sheets are apparent in the Fig. 6a. Another three current sheets appear later and are to some extent influenced by the more evolved flow patterns of the former two current sheets. The evolution of the most developed current sheets in Fig. 6a resembles very closely to that of Fig. 3a, i.e. the magnetic reconnection forms helical flux 

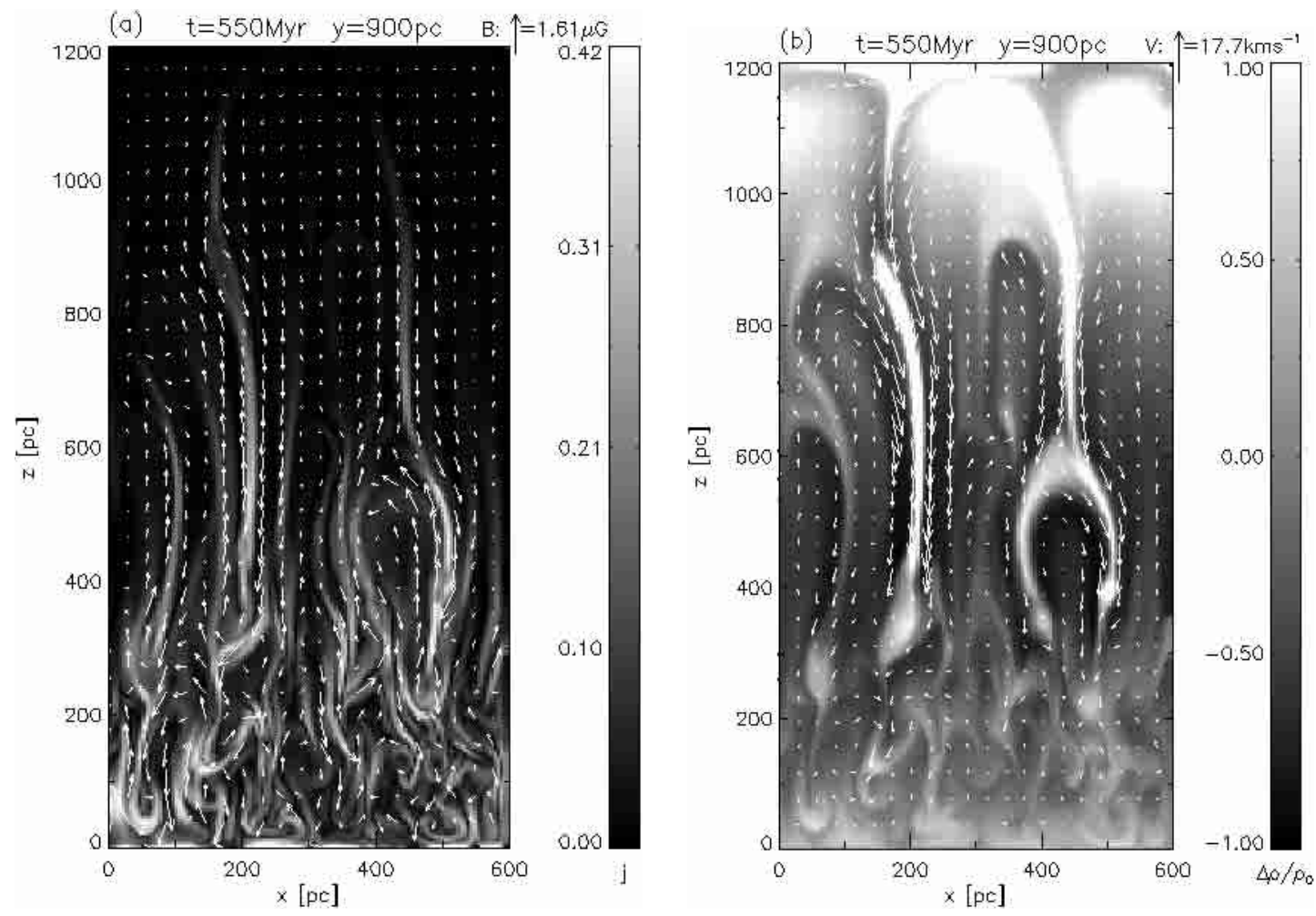

Fig. 7. Vertical cuts in the plane $x z$ and $y=900 \mathrm{pc}$ for the run D1 at $t=550$ Myr. Arrows represent magnetic field a) and velocity b). Shades indicate the current density a) and mass density b).
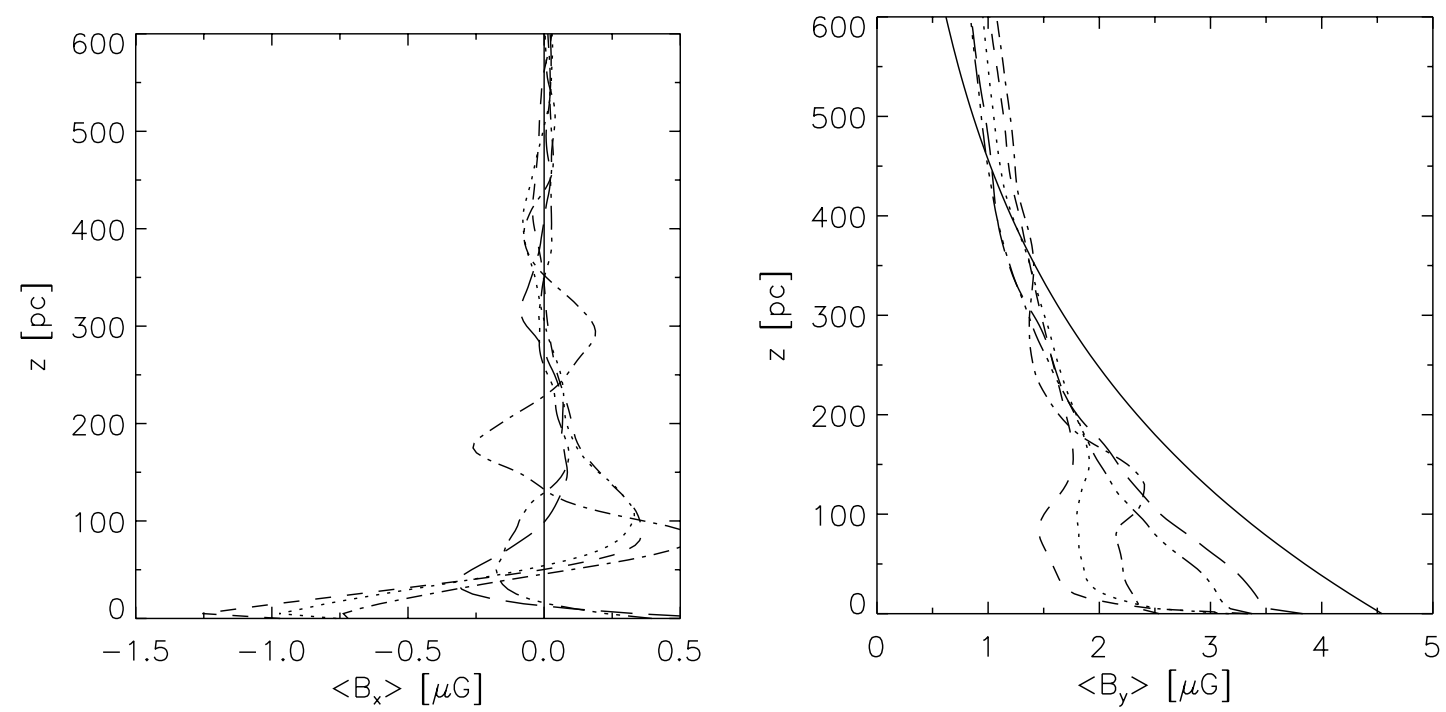

Fig. 8. The mean magnetic field (averaged over planes $z$ = const.) in the $x$-direction a) and $y$-direction b) for different runs and times corresponding to the maximum of $\left\langle B_{x}\right\rangle$ for each run. The full line denotes the magnetic field distribution for $t=0 \mathrm{Myr}$ (the same for all runs). The remaining lines correspond to following runs: dashed $-\mathrm{B} 0$ at $t=500 \mathrm{Myr}$, dotted $-\mathrm{B} 1$ at $t=500 \mathrm{Myr}$, dash-dotted $-\mathrm{A} 1 t=500 \mathrm{Myr}$, long-dash $-\mathrm{C} 1$ at $t=530 \mathrm{Myr}$ and dash-triple-dot $-\mathrm{D} 1$ at $t=550 \mathrm{Myr}$.

tubes surrounding elongated gas condensations. A second pair of helical flux tubes continues to form above the first pair placed at $z \simeq 100 \mathrm{pc}$ and the gas is supplied to the flux tubes from vertical gas sheets above.

A similar evolution is observed in the run B1 shown in Fig. 4a. In this case two out of three current sheets develop earlier and then produce helical flux tubes on two levels. Because the flux tubes contain the gas collected before reconnection, they are heavier than the surroundings and start to move toward the galactic midplane the bottom of the computational box in our simulations. Different tubes become packed tightly in lower parts of the box and subsequently form current sheets in between each other. The implied reconnection merges neighboring flux tubes and leads to the formation of large-scale poloidal magnetic field in the $x$-direction at $t=700 \mathrm{Myr}$ in the 


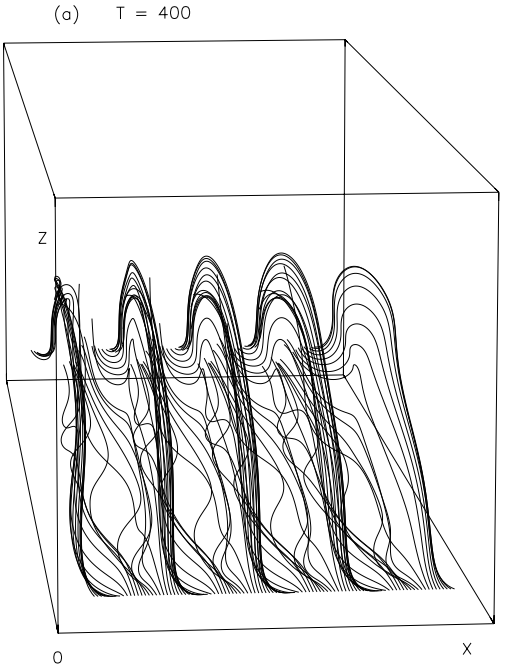

(b) $T=500$

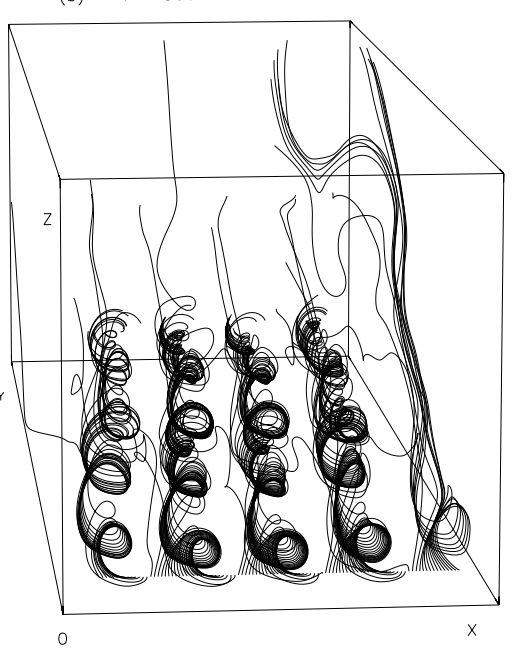

(c) $T=700$

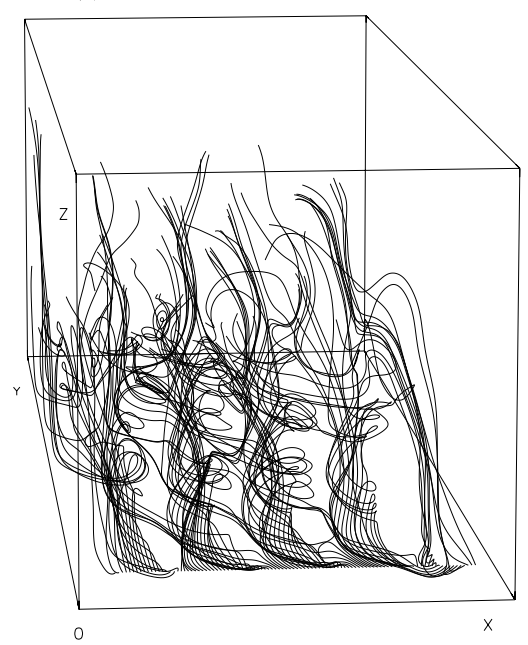

Fig. 9. Topology of magnetic field lines at three time steps for the runs A0 and A1. At $t=400 \mathrm{Myr}$ magnetic filed lines represent a wavelike deformation typical for the Parker instability in the run A0 and in the run A1 before the magnetic reconnection starts to operate a). The helical magnetic tubes seen at $t=500 \mathrm{Myr}$ in the run $\mathrm{A} 1$ result from the magnetic reconnection b). The subsequent coalescence of flux tubes in the run A1 produces the large scale poloidal magnetic field seen at $t=700$ Myr c). same way as in the case of the run A1 (see Figs. 3b, 4b and 5).

In the opposite limit of the large transverse dominating wavenumber - the case D1 (see Fig. 7a) a large number of closely packed current sheets develop. As in previous cases magnetic reconnection leads to the formation of helical flux tubes surrounding gas filaments, however in the present case the tubes as well as the filaments are much thinner and their persistence is much more transient. We show a complementary plot of density and velocity in Fig. 7b. The lower part of the density and velocity plot up to $z \sim 300 \mathrm{pc}$ looks different than the upper parts. The density distribution looks rather irregular, presenting only weak signatures of gas sheets and filaments with small spatial variability scale. That difference can be explained by the fact that the system of current sheets has developed earlier in the lower part of the simulation volume. As a result, the reconnection changed significantly the magnetic field topology and the flow pattern became more turbulent. On the other hand the upper parts $z \geq 300$ pc display regular gas downflows along the sheets toward the emerging gas filaments. It is noticeable that the dominating scale of the developed perturbation grows with $z$. To explain this we note that although the modes of larger perpendicular wavelengths grow slower, they are less dissipative and finally win the competition with the short perpendicular wavelength modes.

The mean value and orientation of the resulting magnetic field is dependent on $z$ as well as on the characteristics of the initial perturbation. In order to present how much of the poloidal magnetic field is produced by the Parker instability in the presence of the Coriolis force we average the magnetic field over planes $z=$ const. and present the mean values of $\left\langle B_{x}(z)\right\rangle$ and $\left\langle B_{y}(z)\right\rangle$ in Fig. 8 for different runs. The full lines denote the magnetic field distribution for $t=0$ (the same for all runs). The other curves defined in the figure caption represent the vertical profile of the mean horizontal magnetic field for the moments of time when the mean value $<B_{x}(z)>$ reaches its maximum at some $z$. It is apparent in Fig. 8a that the largest values of the mean magnetic field $<B_{x}(z)>$ are reached for the runs with small transverse horizontal wavenumbers in the perturbation and that the amount of $\left\langle B_{x}(z)\right\rangle$ decreases with the growth of the transverse wavenumber.

Let us note that $\left\langle B_{x}(z)>\sim 1.25 \mu \mathrm{G}\right.$ for the run B0 and $\left(<B_{x}(z)>\sim 1.00 \mu \mathrm{G}\right)$ for B1 close to the midplane. The smaller $\left\langle B_{x}>\right.$ for the resistive case B1 in comparison to $\mathrm{B} 0$ is perhaps related to the fact that the reconnection changes the flow pattern and through the formation of flux tubes and limits the flow speeds entering the Coriolis force. The same effect may explain the smaller amount of $\left\langle B_{x}(z)>\right.$ for the large transverse wavenumber perturbations, which lead to faster development of current sheets. Some other effects leading to the limitation of $<B_{x}(z)>$ should be also considered. In the case of small horizontal wavenumbers the freedom for the gas motions in the $x$ directions is somewhat related to the transverse 
horizontal wavenumber: the displacements of gas and the frozen in magnetic field $\Delta x$ are of the order of a single wave period $\lambda_{x}=2 \pi / k_{x}$ in that direction. Since the parallel wavelength $\lambda_{y}=2 \pi / k_{y}$ is limited from below by the magnetic tension stabilizing the small $\lambda_{y}$ components of the perturbation, the characteristic horizontal pitch angle of magnetic field lines $\sim \lambda_{x} / \lambda_{y}$ with respect to the $x$-axis has to be smaller for larger $k_{x}$.

It is interesting that the distribution of $\left\langle B_{x}(z)\right\rangle$ splits runs in two groups. For some runs (B0, B1, A1) $<B_{x}>$ is negative close to the midplane and for the other runs (C1, D1 it is positive. We note that the negative $<B_{x}>$ appear close to the midplane for cases with small transverse wavenumbers and positive $\left.<B_{x}\right\rangle$ close to the midplane appears for larger transverse wavenumbers. Our hypothesis is that the low transverse wavenumber perturbations lead to heavier gas filaments which are more efficiently pressed to the plane $z=0$ than the light filaments appearing for large transverse wavenumbers (compare Figs. 3 and 4 with Figs. 6 and 7). Although the sign of the magnetic helices is the same in all cases, the heavy flux tubes gathered at lower layers of the simulation box contribute to $\left\langle B_{x}\right\rangle$ more coherently, while in the case of larger transverse wavenumbers the medium underlying the helical tubes is twisted in the opposite direction. The simulation A1 is an intermediate case. For this run $\left\langle B_{x}\right\rangle$ is initially positive and later on it becomes negative as the gas filaments surrounded by the helical magnetic field fall down.

The most significant property of our model is the production and separation in $z$ of the oppositely directed components of $\left\langle B_{x}\right\rangle$. We note that production of the mean poloidal magnetic field is accompanied by the vertical upward transport of the magnetic flux, which is apparent in Fig. $8 \mathrm{~b}$ for all the runs. It is characteristic that the amount of generated $<B_{x}>$ up to $z \sim 300$ pc correlates with the amount of $\left\langle B_{y}\right\rangle$ which is transported to upper layers of the disk. This separation associated with the net vertical upward transport of magnetic flux represents a behavior which is required by the dynamo process. Strictly speaking we need to add the differential rotation to regenerate and amplify the magnetic field which is transported out of the disk by the Parker instability. Without the differential rotation and with our present choice of simulation parameters we do not observe any growth of the magnetic energy within the simulation volume. The differential rotation increases, however, significantly the level of complexity of the problem and will be incorporated in a separate paper.

\section{Summary and discussion}

We considered the general problem of plasma reactions to external disturbances performing a series of numerical simulations of the Parker instability in both the ideal and the resistive MHD cases. In the ideal cases we studied the Parker instability in an ideal rotating plasma, i.e. in which the electrical resistivity is zero. In this configuration only slow topological changes associated with the numerical resistivity are observed. In the second series of simulations we introduced the well-known concept of anomalous resistivity (Ugai 1992; Tanuma et al. 1999; Konz et al. 2000), which is spatially dependent on the value of the current density. Above a critical value of the current density, magnetic reconnection sets in and is accompanied by drastic topological changes and dissipation of magnetic fields on small spatial scales. The numerical resistivity results in topological changes as well, however in the present setup it operates much slower than the explicitly introduced current-dependent resistivity.

The initially homogeneous magnetic field unstable with respect to Parker instability now develops in a welldefined flux tube structure. Under the action of magnetic reconnection the topology of magnetic field evolves and helically twisted flux tubes are formed. On a longer time period these flux tubes form the next generation of current sheets leading to the flux tube coalescence. The final state contains a substantial amount of the large scale, $z$-dependent poloidal magnetic field component. A similar scenario of formation of the poloidal magnetic field through the interaction of helical structures of the magnetic field obtained from the Parker instability has been proposed by Hanasz \& Lesch (1998). The poloidal magnetic field resulting from the described processes can be identified with a spiral magnetic field structure in galactic disks. We suggest that such an evolution of unstable galactic magnetic fields can be a part of the $\alpha \omega$ fast dynamo of the kind proposed by Parker (1992).

Formation of a spiral and filamentary magnetic field structure has been also observed in the ideal MHD simulations of Parker-unstable magnetized accretion disks by Machida et al. (2000). These authors report the formation of numerous small current sheets in the disk. Although the magnetic reconnection is not explicitly incorporated in their simulations, the observed transformation from toroidal to spiral structure of magnetic fields indicates that some topological evolution takes place due to the numerical resistivity.

We note that the topological evolution of galactic magnetic fields may be much more sophisticated if only the cosmic rays are taken into account. The formation of helical flux tubes will inevitably influence the propagation of cosmic rays. One can expect that cosmic rays produced in supernova remnants and injected into the flux tubes will imply their instability and buoyant rise in a way described by Hanasz \& Lesch (2000). As we have shown in our 1997 paper (Hanasz \& Lesch 1997, see Fig. 8 and related text) the behavior of the Parker-shearing instability changes qualitatively depending on the relative strength of the shearing and the buoyancy forces. It resembles the Parker instability if the buoyancy is strong enough compared to the shearing force, whereas in the opposite case it reproduces the development of the shearing instability. The strong contribution of the cosmic rays to the buoyancy then implies that the dominance of the Parker instability behavior can be expected even in the presence of differential shear. Furthermore, since cosmic 
rays significantly shorten the timescale of the Parker instability we expect a very efficient dynamo process in the presence of differential rotation (Hanasz \& Lesch 1997, 1998).

Finally, we would like to emphasize the importance of the non-ideal treatment of a globally ideal magnetized plasma. Although on large spatial scales the resistivity due to Coulomb collisions may be negligible and the plasma can be treated as ideal, any disturbance of the magnetized plasma will lead to localized dissipation regions simply because the current density in an ideal plasma will rise until it is so strong that plasma oscillations are excited, which locally provide an anomalous resistivity. This evolution is natural for any ideal system, which always has the tendency to avoid any kind of "crisis" by shifting strong gradients into small localized boundary layers. In the case of an ionized gas these boundary layers correspond to current sheets in which the deviation from idealness is provided by the intrinsic mechanisms which belong to phasespace instabilities exciting strong fluctuations of plasma density, i.e. strong electromagnetic oscillations limited to small spatial scales. These deviations from idealness are responsible for the localized dissipation which are accompanied by global topological changes of the magnetic field into a flux tube structure. This may appear in all kinds of magnetized ideal astrophysical plasmas exposed to external distortions or instabilities.

Acknowledgements. $\mathrm{MH}$ thanks Alexander von Humboldt Foundation for the AvH Fellowship supporting the work in this paper and the München University Observatory, for the kind hospitality. This work was partly supported by the Polish Committee for Scientific Research (KBN) through the grants PB 457/P03/97/13 and PB 4264/P03/99/17. We thank the Laboratory for Computational Astrophysics, University of Illinois for the kind permission to use the ZEUS3D MHD code, Grzegorz Kowal for his help on applying the software. The computations were partially performed on the Convex-SPP machines at the Academic Computer Centre "Cyfronet" in Kraków (grants No. KBN/C3840/UJ/011/1996 and $\mathrm{KBN} / \mathrm{SPP} / \mathrm{UJ} / 011 / 1996)$.

\section{References}

Beck, R. 2001, Space Sci. Rev., in press

Birk, G. T., Lesch, H., \& Neukirch, T. 1998, MNRAS, 296, 165 Biskamp, D. 1997, Nonlinear Magnetohydrodynamics (Cambridge University Press), 173

Braginskii, S. I. 1965, Rev. of Plasma Physics, 1, 205

Brandenburg, A., \& Schmitt, D. 1998, A\&A, 338, L55
Chyży, K., Beck, R., Kohle, S., Klein, U., \& Urbanik, M. 2000, A\&A, 355, 128

Dalgarno, A., \& McCray, R. A. 1972, ARA\&A, 10, 375

Ferriere, K. 1998a, ApJ, 497, 759

Ferriere, K. 1998b, ApJ, 503, 700

Fleming, T. P., Stone, J. M., \& Hawley, J. F. 2000, ApJ, 530, 464

Foglizzo, T., \& Tagger, M. 1994, A\&A, 287, 297

Foglizzo, T., \& Tagger, M. 1995, A\&A, 301, 293

Hanasz, M., \& Lesch, H. 1993, A\&A, 278, 51

Hanasz, M., \& Lesch, H. 1997, A\&A, 321, 1007

Hanasz, M., \& Lesch, H. 1998, A\&A, 332, 77

Hanasz, M., \& Lesch, H. 2000, ApJ, 543, 235

Hawley, J. F., Gammie, C. F., \& Balbus, S. A. 1996, ApJ, 464, 690

Kahn, F. D., \& Brett, L. 1993, MNRAS, 263, 37

Konz, Ch., Wiechen, H., \& Lesch, H., \& PoP, 7, 5159

Kim, J., Hong, S. S., Ryu, D., \& Jones, T. W. 1998, ApJ, 506, L139

Kim, J., Ryu, D., \& Jones, T. W. 2001, ApJ, 557, 446

Kim, W.-T., \& Ostriker, E. C. 2000, ApJ, 540, 372

Lachièze-Ray, M., Assèo, E., Cesarsky, C. J., \& Pellat, R. 1980, ApJ, 238, L175

Machida, M., Hayashi, M. R., \& Matsumoto, R. 2000, ApJ, 532, L67

Moss, D., Shukurov, A., \& Sokoloff, D. 1999, A\&A, 343, 120

Otmianowska-Mazur, K., Chyży, K. T., Soida, M., \& von Linden, S. 2000, A\&A, 359, 29

Parker, E. N. 1966, ApJ, 145, 811

Parker, E. N. 1967, ApJ, 149, 535

Parker, E. N. 1979, Cosmical Magnetic Fields (Oxford)

Parker, E. N. 1992, ApJ, 401, 137

Parker, E. N. 1994, Spontaneous Current Sheets in Magnetic Fields (Oxford)

Parker, E. N., \& Jokipii, J. R. 2000, ApJ, 536, 331

Priest, E. R. 1987, Solar Magnetohydrodynamics (Reidel, Dordrecht)

Raymond, J. C. 1992, ApJ, 384, 502

Reynolds, R. J., Haffner, L. M., \& Tufte, S. L. 1999, ApJ, 525, L21

Sagdeev, R. Z. 1979, Rev. Mod. Phys., 51, 1

Stone, J. M., \& Norman, M. L. 1992a, ApJS, 80, 753

Stone, J. M., \& Norman, M. L. 1992b, ApJS, 80, 791

Tanuma, S., Yokoyama, T., Kudoh, T., et al. 1999, PASJ, 51, 161

Tanuma, S., Yokoyama, T., Kudoh, T., \& Shibata, K. 2001, ApJ, 551, 312

Terquem, C., \& Papaloizu, J. C. B. 1996, MNRAS, 279, 767

Treumann, R. A., \& Baumjohann, W. 1997, Advanced Space Plasma Physics (Imperial College Press, London)

Ugai, M. 1992, Phys. Fluids B, 4, 2953

Zweibel, E. G., \& Kulsrud, R. M. 1975, ApJ, 201, 63 